\title{
Finasterida tópica y alopecia androgenética masculina y femenina
}

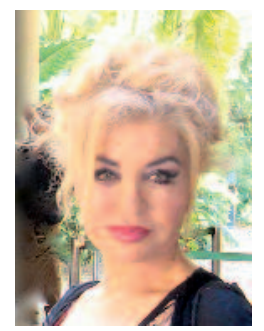

Aurora Guerra Tapia

Profesora titular de Dermatología. Departamento de Medicina.

Facultad de Medicina.

Universidad Complutense de Madrid.

Jefa de Sección de Dermatología. Hospital Universitario

12 de Octubre. Madrid. Profesora de Dermatología. Universidad Internacional de La Rioja (UNIR).

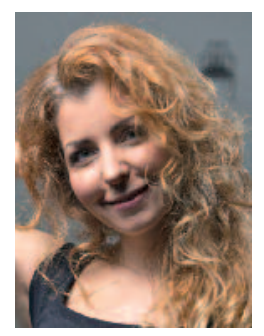

\section{Elena González-Guerra}

Profesora asociada de Dermatología. Departamento de Medicina.

Facultad de Medicina.

Universidad Complutense de

Madrid.

Médico adjunto.

Servicio de Dermatología.

Hospital Clínico San Carlos. Madrid.

Profesora de Dermatología.

Universidad Internacional

de La Rioja (UNIR).
No es la primera vez que vamos a referirnos a la finasterida como tratamiento de la alopecia. Lo hemos hecho previamente exponiendo ponencias en foros científicos nacionales e internacionales, como son los congresos y reuniones de la Academia Española de Dermatología y Venereología (AEDV) o del Colegio Íbero-Latinoamericano de Dermatología (CILAD), y escribiendo artículos de diferentes tipos en revistas acreditadas y divulgativas ${ }^{1-3}$.

La finasterida ha merecido atención en dermatología por ser un potente antiandrógeno no esteroideo, que inhibe la $5 \alpha$-reductasa de tipo 2 , evitando, de este modo, la conversión de testosterona libre a $5 \alpha$-DHT

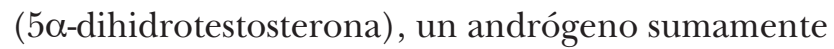
potente, que desempeña un importante papel en la etiopatogenia de la alopecia androgenética en hombres y mujeres.

En el varón, la $5 \alpha$-reductasa de tipo 2 se encuentra predominantemente en la próstata y en determinadas regiones del folículo piloso, por lo que su acción resulta altamente específica y con escasos efectos secundarios, que, por otra parte, suelen remitir espontáneamente, incluso, con la continuidad del tratamiento. En cuanto a la eficacia, se ha comprobado que, en el $83 \%$ de los varones que toman finasterida en dosis de $1 \mathrm{mg}$ al día, se detiene la miniaturización del cabello e, incluso, en el $66 \%$ de los casos, aumenta su cantidad. Estas afirmaciones son asumidas por la comunidad científica, apoyada tanto en los ensayos clínicos como en los ya largos años de uso, de tal forma que es el único medicamento oral aprobado por la Food and Drug Administration estadounidense para el tratamiento de la alopecia masculina.

En la mujer, la finasterida se considera un antiandrógeno eficaz en dosis más altas que en el varón ${ }^{4}$. Sin embargo, el problema se complica por su poder teratógeno en caso de embarazo. La finasterida es 
un medicamento de categoría X, por lo que está contraindicado en mujeres en edad fértil si no usan métodos anticonceptivos seguros. Queremos destacar que son muchos los medicamentos con esta categorización que, sin embargo, se usan en la mujer con las medidas anticonceptivas correspondientes. Véase la isotretinoína para el acné, el metotrexato para la psoriasis, el diclofenaco para la inflamación articular, o el sedante flurazepam, entre otros. Parece, por lo tanto, que la demonización o no del uso femenino de la finasterida solo depende de un manejo correcto y una indicación necesaria.

Aun así, siempre resulta deseable utilizar los principios activos aprobados en las enfermedades cutáneas de forma tópica, evitando el proceso metabólico sistémico y las posibles interacciones, y minimizando los riesgos e, incluso, el componente psicológico que parece rotular con negrita el diagnóstico de una enfermedad cuando se requiere medicación oral frente a la que solo precisa terapia tópica.

Con esta premisa, y dada la gran prevalencia de la alopecia androgenética en hombres y mujeres, no es raro que el interés por el uso tópico de la finasterida se haya desbordado en los últimos tiempos.

Introduciendo en cualquier buscador de Internet las palabras «finasterida tópica», aparecen en segundos más de 100000 entradas. No ocurre lo mismo en las bases bibliográficas médicas, donde las referencias son escasas.

Las primeras investigaciones publicadas al respecto buscaron estabilizar la molécula mediante determinados tensoactivos in vitro y determinar qué excipientes favorecen la liberación de la dosis deseada. Los siguientes pasos confirmaban los hallazgos previos, esta vez, sobre piel de animales (oreja de cerdo) y piel de cadáver. Ya in vivo, se comprobó la penetración transcutánea del principio activo en ratas sin pelo y, un tiempo después, se evaluó la farmacodinamia y disminución de la DHT sérica y en el cuero cabelludo a la semana de tratamiento en humanos, de forma com- parativa con la finasterida oral, y con resultados menores.

Pero ¿podemos decir que un tratamiento ha sido probado como efectivo si no se apoya en estudios doble ciegos controlados con placebo? Obviamente, estos estudios son necesarios para confirmar sin dudas la eficacia clínica de cualquier tratamiento y, en el caso de la finasterida de uso tópico, también.

Hoy por hoy, tenemos escasa evidencia de alto valor de la eficacia de la finasterida tópica, y ninguna en mujeres con alopecia, aunque sí la hay en el tratamiento del hirsutismo, al que no nos referiremos, por no ser objeto de nuestro editorial.

En 2009, se publica un estudio doble ciego que compara 45 pacientes separados en dos grupos tratados durante 23 meses con finasterida oral y placebo tópico el primero, y finasterida tópica en gel y placebo oral el segundo, encontrando una eficacia similar en ambos casos ${ }^{5}$. El segundo estudio doble ciego se publica en 2012, y compara la finasterida tópica al 0,1\% y el minoxidil al 3\% con minoxidil al $3 \%$ solo, no encontrando diferencias entre ambos ${ }^{6}$. Y poco más. Algunas experiencias de expertos tricólogos, que refieren buenos resultados, pero sin demostraciones objetivas.

¿Qué podemos concluir?

Que el interés existe, que la esperanza parece sustentada tanto en las escasas investigaciones básicas como en las clínicas, pero que todavía no podemos tener resultados definitivos. Pronto los tendremos, ya que somos varios los grupos de investigación tricológica que estamos participando actualmente en un nuevo ensayo doble ciego, controlado con placebo, multicéntrico, solo en varones, del que esperamos datos concluyentes.

¿Y en la mujer?

Seguiremos esperando.

\section{BIBLIOGRAFÍA}

1. Guerra-Tapia A, Bruña V, Delgado AM. Finasterida y alopecia de la mujer, 4 años después. Más Dermatol. 2011;(13): 21-4. 
2. Rivera R, Guerra-Tapia A. Management of androgenetic alopecia in postmenopausal women. Actas Dermosifiliogr. 2008;99(4):257-61.

3. Rivera R, Guerra Tapia A. ¿Sirve la finasterida en la alopecia de la mujer? Más Dermatol. 2007;(1):21-4.

4. Camacho-Martínez FM. Hair loss in women. Semin Cutan Med Surg. 2009;28(1):19-32.

5. Hajheydari Z, Akbari J, Saeedi M, Shokoohi L. Comparing the therapeutic effects of finasteride gel and tablet in treatment of the androgenetic alopecia. Indian J Dermatol Venereol Leprol. 2009;75(1):47-51.

6. Tanglertsampan C. Efficacy and safety of $3 \%$ minoxidil versus combined $3 \%$ minoxidil / $0.1 \%$ finasteride in male pattern hair loss: a randomized, double-blind, comparative study. J Med Assoc Thai. 2012;95(10):1312-6. 